\title{
Two-way DF relaying assisted D2D communication: ergodic rate and power allocation
}

\author{
Yiyang $\mathrm{Ni}^{1^{*}}$, Yuxi Wang ${ }^{1}$, Shi Jin ${ }^{2}$, Kai-Kit Wong ${ }^{3}$ and Hongbo Zhu ${ }^{4}$
}

\begin{abstract}
In this paper, we investigate the ergodic rate for a device-to-device (D2D) communication system aided by a two-way decode-and-forward (DF) relay node. We first derive closed-form expressions for the ergodic rate of the D2D link under asymmetric and symmetric cases, respectively. We subsequently discuss two special scenarios including weak interference case and high signal-to-noise ratio case. Then we derive the tight approximations for each of the considered scenarios. Assuming that each transmitter only has access to its own statistical channel state information (CSI), we further derive closed-form power allocation strategy to improve the system performance according to the analytical results of the ergodic rate. Furthermore, some insights are provided for the power allocation strategy based on the analytical results. The strategies are easy to compute and require to know only the channel statistics. Numerical results show the accuracy of the analysis results under various conditions and test the availability of the power allocation strategy.
\end{abstract}

Keywords: Device-to-device communication, Two-way relay, Decode-and-forward, Ergodic rate, Power allocation

\section{Introduction}

In recent years, a seamless increase of various innovative multi-media services has kept pushing the limits of current wireless networks, urging for higher-speed communications $[1,2]$. As the smart mobile devices popularize progressively, the demand for higher wireless transmission rate will grow exponentially in the next decades and a 1000 times increase in the current system capacity is required by $2020[3,4]$. However, the fourth generation mobile communication systems which can support a rate of $1 \mathrm{Gbit} / \mathrm{s}$ hardly satisfy the demand of mobile communication in the next 10 years [5]. Therefore, the fifth generation (5G) cellular network is being considered [6]. One of the key technologies for $5 \mathrm{G}$ systems, which has recently attracted huge attention and can greatly enhance the spectral efficiency [7-9], is device-to-device (D2D) communication.

Generally speaking, there are two main types of radio resource sharing methods used by $\mathrm{D} 2 \mathrm{D}$ communication in

\footnotetext{
*Correspondence: niyy@jssnu.edu.cn

1 Jiangsu Normal University, Nanjing 210013, China

Full list of author information is available at the end of the article
}

a cellular network: underlay and overlay inband communication [10]. For the overlay case, D2D communication takes part of the cellular resources and leaves the other half to the cellular user. Therefore, there is no interference between cellular link and D2D link. In contrast, for the underlay case, D2D users use the same resources with the cellular user causing interference to both the cellular link and D2D link, and the base station (BS) is required to coordinate the transmit power for both the cellular and D2D users [11-13]. Compared with the conventional cellular communication, obviously, comparing to the traditional cellular communication, D2D communication can save the resources and improve the throughput greatly, due to the fact that only half of the resources are used [14].

Although lots of advantages can be taken by D2D communication, it also faces a rigorous challenge-the inter-channel interference [15]. To reduced ICI from the D2D links, interference management and coordination is important for physical-layer designs, e.g., power control and resource allocation. Great efforts to tackle the interference problem have been made in [16-18]. For

\section{Springer Open}

(c) The Author(s). 2017 Open Access This article is distributed under the terms of the Creative Commons Attribution 4.0 International License (http://creativecommons.org/licenses/by/4.0/), which permits unrestricted use, distribution, and reproduction in any medium, provided you give appropriate credit to the original author(s) and the source, provide a link to the Creative Commons license, and indicate if changes were made. 
traditional D2D communication systems, many power allocation strategies have presented to improve the performance of cellular link or D2D link. For example, in [12], a joint power allocation scheme was proposed which maximizes the sum-rate of D2D link and cellular link. The authors in [13] have also given an interference-aware channel allocation scheme based on Hungarian algorithm to maximize the number of permitted D2D communication pairs. In order to effectively guarantee the cellular service, Yu et al. [16] proposed a transmit power allocation algorithm while the authors in [18] presented an optimum resource allocation and power control between the cellular and D2D link to improve the performance of D2D communication.

Equally, relay-assisted communication in cellular networks has also demonstrated great potential in enhancing the system performance [19-21]. Efforts have been firstly spent on analyzing the one-way relay-assisted systems with different relay protocols including amplify-andforward (AF), decode-and-forward (DF), and compressand-forward (CF) protocols, e.g., [22-24]. Relaying technologies have also been proposed for two-way communications $[25,26]$. The transmission schemes over two, three, or four time slots for two-way relay protocols have been investigated in [27]. Recently, the application of relay techniques in conjunction with D2D communication has increasing interest as a means of achieving further performance improvements and coverage enhancement in cellular networks. Also, the D2D communication assisted by a relay node can alleviate the interference to the cellular link as it can reduce the transmit power of the D2D users. A comprehensive resource allocation framework for the one-way DF relay-assisted D2D communication was presented in [28]. In [29], the authors presented a joint relay selection and power allocation scheme for oneway AF relayed D2D communication. Based on the outage probability performance, Wang et al. [30] presented an interference constrained precondition for the one-way DF relay aided D2D communication. It is obvious that the combination of the D2D communication and the relayassisted communication is a very effective method to improve the performance of the next-generation cellular system. One-way relay-assisted system has been investigated in [31-33] which presented closed-form expressions for the system performance. However, it needs four time slots to realize a complete communication with one-way relay policy while only two time slots are required for the two-way relay policy. Due to its potential to enhance the spectrum efficiency, two-way relay policy has attracted a great deal of attention. An optimal power allocation algorithm was provided to minimize the outage probability of D2D users for the two-way AF relay-assisted D2D communication system [34]. However, the interference was not considered. Sun et al. [35] proposed a security-embedded interference avoidance scheme to improve the system error performance in which the D2D user served as relays to assist the two-way transmission between two cellular users. In [36], authors proposed a new scheme in which three-phase D2D communication assisted with two-way DF relay is considered. The Pareto boundary of the region of the sum rate of D2D link versus that of the cellular link was found by optimizing the transmit power at base station and cellular user, as well as, D2D users [37].

Motivated by the interest of two-way relay-assisted system, as our major contribution, we will derive closed-form expressions of the ergodic rate for the proposed relayassisted strategy, and investigate the effects of noise, the predetermined ratio, the received power of signals including the desired signal, and the interference. Two cases in which the relaying terminal has the same (symmetric) and different (asymmetric) received power from the two terminals exchanging information are considered. We then study the performance for the weak interference case and high signal-to-noise ratio (SNR) case. The approximations of ergodic rate for these cases are derived respectively. Based on these approximations, we present closed-form power allocation strategies which involve several key system coefficients and can be easily implemented. Numerical results confirm the accuracy of the analysis results and show the tightness of our approximate results. The improvement of the ergodic rate by using the power allocation strategy also can be found from the numerical results. To the best of our knowledge, this is the first analytical result that is applicable for D2D communication with two-way DF relaying.

\section{System model}

We consider a D2D communication system assisted with a two-way DF relay node. We assume that the inter-channel interference between the users can be properly controlled by some efficient controlling mechanism. We focus on the interference between D2D link and cellular link, which is caused by sharing the same resources. Consider an isolated cell scenario as shown in Fig. 1 where one base station, one cellular user and a pair of D2D users are present (i.e., UEC, $\mathrm{UED}_{1}$ and $\mathrm{UED}_{2}$ ). We assume the $\mathrm{BS}$ and all the users are equipped with single antenna. $U_{E D}$ and $U_{E D}$ exchange the messages with each other via UEr employing two-way DF protocol. We denote the transmit power of terminal $i$ as $P_{i T}$ for $(i=1,2, r, c, b)$ which, respectively, refers to $\mathrm{UED}_{1}, \mathrm{UED}_{2}$, UEC, the BS and the relay. For fading channels, we use $h_{i j}$ to denote the Rayleigh fading channel coefficients of $i-j \operatorname{link}($ for $j=1,2, r, c, b$ ). It is assumed that the noise $n_{i}$ is an independently and identically distributed complex Gaussian noise with zero mean and $N_{0}$ variance. The received power $P_{i j}$ at user $j$ measured at $d_{i j}$ away from the transmitter $i$ is defined as 


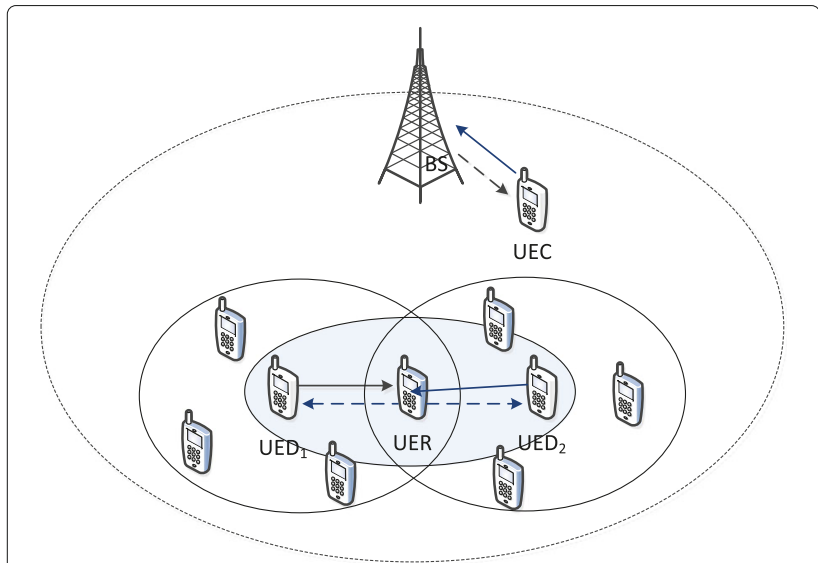

Fig. 1 A D2D communication aided by two-way DF relaying underlaying cellular network

$$
P_{i j}=P_{i T} d_{i j}^{-\alpha},
$$

where $\alpha$ denotes the path-loss exponent. We assume that the uplink and downlink channels are reciprocal, invariant, and formatted in two consecutive equal time slots. As shown in Fig. 2 (a), during the first time slot, $U D_{1}$ and UED 2 transmit messages $S_{1}$ and $S_{2}$ to UEC simultaneously. At the same time, UEC sends messages to the BS. Thus UER is effected by the noise plus the interference from cellular link. The desired signal received at the relay node can be given by

$$
y_{r}^{D F}=\sqrt{P_{1 r}} h_{1 r} S_{1}+\sqrt{P_{2 r}} h_{2 r} S_{2}+\sqrt{P_{c r}} h_{c r} S_{c}+n_{r} .
$$

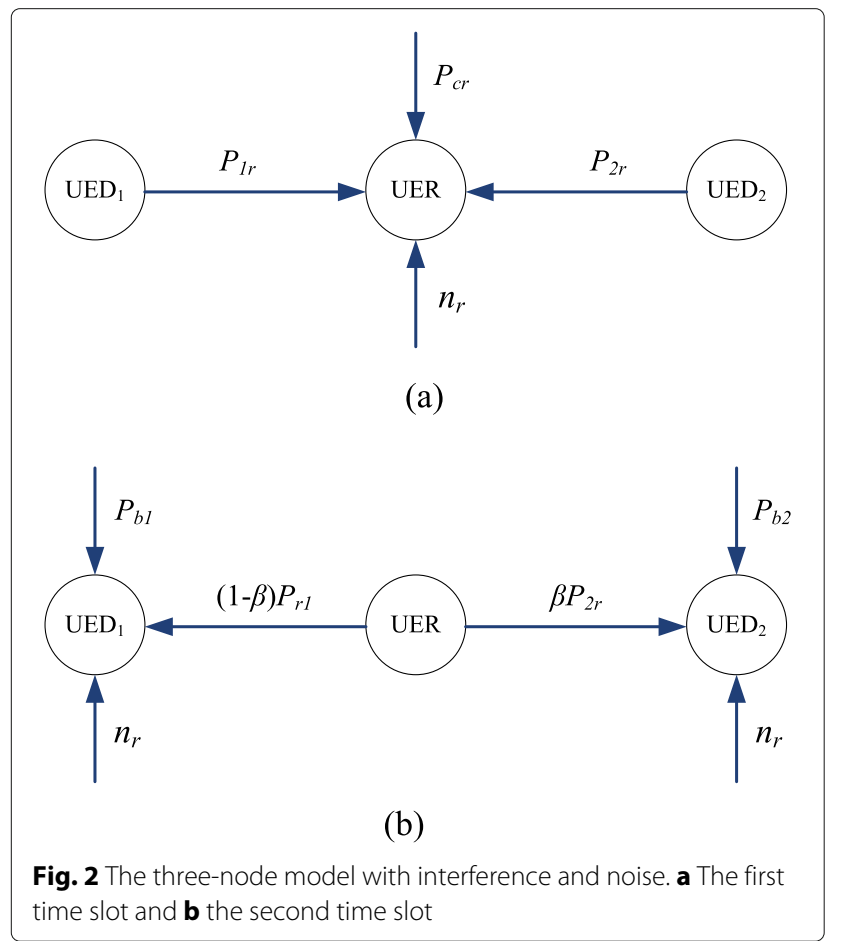

As shown in Fig. 2 (b), after the decoding process of $y_{r}^{D F}$, UER decodes $S_{1}$ and $S_{2}$ and transmits the message $S_{r}$ to $U D_{1}$ and $U E D_{2}$ in the second time slot that [38]

$$
S_{r}=\sqrt{\beta} S_{1}+\sqrt{1-\beta} S_{2},
$$

where the relay uses an average transmit power of $\beta P_{r T}$ for the forward direction and $(1-\beta) P_{r T}$ for the backward direction. Besides, the BS sends the messages to UEC simultaneously. After the self-interference cancelation procedure, the received signal at the $\mathrm{UED}_{1}$ and $\mathrm{UED}_{2}$ can be written as follows, respectively,

$$
y_{1}^{D F}=\sqrt{P_{r 1}(1-\beta)} h_{r 1} S_{2}+\sqrt{P_{b 1}} h_{b 1} S_{b}+n_{1},
$$

and

$$
y_{2}^{D F}=\sqrt{P_{r 2} \beta} h_{r 2} S_{1}+\sqrt{P_{b 2}} h_{b 2} S_{b}+n_{2} .
$$

Having established the signal model in (2)-(5), we now present the expressions of the signal-to-interference-plusnoise ratio (SINR) for different links. For the first phase, according to the received signals at UER in (2), we can obtain the SINR for the UED 1 -UER link and $\mathrm{UED}_{2}-$ UER link which can be respectively expressed as

$$
\gamma_{1 r}=\frac{P_{1 r}\left|h_{1 r}\right|^{2}}{P_{c r}\left|h_{c r}\right|^{2}+N_{0}}
$$

and

$$
\gamma_{2 r}=\frac{P_{2 r}\left|h_{2 r}\right|^{2}}{P_{c r}\left|h_{c r}\right|^{2}+N_{0}} .
$$

The sum-SINR at the relay node can be further expressed as [39, 40]

$$
\gamma_{M A C}=\frac{P_{1 r}\left|h_{1 r}\right|^{2}+P_{2 r}\left|h_{2 r}\right|^{2}}{P_{c r}\left|h_{c r}\right|^{2}+N_{0}} .
$$

For the second phase, we also have the SINRs for the relay $-\mathrm{UED}_{1}$ and the relay $-\mathrm{UED}_{2}$ links as

$$
\gamma_{r 1}=\frac{(1-\beta) P_{r 1}\left|h_{r 1}\right|^{2}}{P_{b 1}\left|h_{b 1}\right|^{2}+N_{0}}
$$

and

$$
\gamma_{r 2}=\frac{\beta P_{r 2}\left|h_{r 2}\right|^{2}}{P_{b 2}\left|h_{b 2}\right|^{2}+N_{0}} .
$$

\section{Ergodic rate analysis}

In this section, new closed-form expressions for the ergodic rate of D2D link aided by two-way DF relay are derived firstly. Symmetric (where the received power at the relay from the two source users is the same) and asymmetric (where the received power is different) cases are considered. We assume channel reciprocity and that the relay node can decode $S_{1}$ and $S_{2}$ without errors. According to the definition in [26], the ergodic rate of the DF protocol is then given by

$$
R_{\text {sum }}^{D F}=\min \left(R_{\text {mac }}, R_{1}(\beta)+R_{2}(1-\beta)\right),
$$


where

$$
\begin{aligned}
R_{\text {mac }} & =\frac{1}{2} E\left\{\log _{2}\left(1+\gamma_{\text {mac }}\right)\right\} \\
R_{1}(\beta) & =\frac{1}{2} \min \left(E\left\{\log _{2}\left(1+\gamma_{1 r}\right)\right\}, E\left\{\log _{2}\left(1+\gamma_{r 2}(\beta)\right)\right\}\right),
\end{aligned}
$$

$R_{2}(\beta)=\frac{1}{2} \min \left(E\left\{\log _{2}\left(1+\gamma_{2 r}\right)\right\}, E\left\{\log _{2}\left(1+\gamma_{r 1}(1-\beta)\right)\right\}\right)$.

We are now ready to derive an analytical expression of (11).

\subsection{Exact analysis}

With the above analysis, we now investigate the closedform expression for the ergodic rate. The case of $P_{1 r} \neq P_{2 r}$ is referred to as the asymmetric case. For the symmetric case, since the received power at relay node from $\mathrm{UED}_{1}$ is equal to that from $\mathrm{UED}_{2}$, we use $P_{d r}$ to indicate the received power at the relay node from the D2D users. The ergodic rate of the D2D link under the two cases are derived and presented in the following theorem.

Theorem 1 The ergodic rate of the D2D communication aided by a DF relay is given by

$$
R_{\text {sum }}^{D F}=\frac{1}{2} \min \left(R_{m a c}, R_{1}+R_{2}\right),
$$

where $R_{1}=\min \left(R_{1 r}, R_{r 2}\right), R_{2}=\min \left(R_{2 r}, R_{r 1}\right)$ with

$$
\begin{aligned}
& R_{1 r}=\frac{P_{1 r}}{P_{1 r}-P_{c r}}\left[e^{\frac{N_{0}}{P_{1 r}}} E_{1}\left(\frac{N_{0}}{P_{1 r}}\right)-e^{\frac{N_{0}}{P_{c r}}} E_{1}\left(\frac{N_{0}}{P_{c r}}\right)\right], \\
& R_{r 2}=\frac{\beta P_{r 2}}{\beta P_{r 2}-P_{b 2}}\left[e^{\frac{N_{0}}{\beta P_{r 2}}} E_{1}\left(\frac{N_{0}}{\beta P_{r 2}}\right)-e^{\frac{N_{0}}{P_{b 2}}} E_{1}\left(\frac{N_{0}}{P_{b 2}}\right)\right],
\end{aligned}
$$

$$
R_{2 r}=\frac{P_{2 r}}{P_{2 r}-P_{c r}}\left[e^{\frac{N_{0}}{P_{2 r}}} E_{1}\left(\frac{N_{0}}{P_{2 r}}\right)-e^{\frac{N_{0}}{P_{c r}}} E_{1}\left(\frac{N_{0}}{P_{c r}}\right)\right],
$$

$$
R_{r 1}=\frac{(1-\beta) P_{r 1}}{(1-\beta) P_{r 1}-P_{b 1}}\left[e^{\frac{N_{0}}{(1-\beta) P_{r 1}}} E_{1}\left(\frac{N_{0}}{(1-\beta) P_{r 1}}\right)-e^{\frac{N_{0}}{P_{b 1}}} E_{1}\left(\frac{N_{0}}{P_{b 1}}\right)\right],
$$

and

$$
R_{m a c}=\left\{\begin{array}{l}
R_{m a c}^{A s y} \text { for the asymmetric case, } \\
R_{m a c}^{S y m} \text { for the symmetric case }
\end{array}\right.
$$

with

$$
\begin{aligned}
R_{m a c}^{A s y}= & \frac{P_{1 r}^{2}}{\left(P_{2 r}-P_{1 r}\right)\left(P_{c r}-P_{1 r}\right)} e^{\frac{N_{0}}{P_{1 r}}} E_{1}\left(\frac{N_{0}}{P_{1 r}}\right) \\
& -\frac{P_{2 r}^{2}}{\left(P_{2 r}-P_{1 r}\right)\left(P_{c r}-P_{2 r}\right)} e^{\frac{N_{0}}{P_{2 r}}} E_{1}\left(\frac{N_{0}}{P_{2 r}}\right) \\
& -\frac{P_{1 r} P_{2 r}-P_{c r}\left(P_{1 r}+P_{2 r}\right)}{\left(P_{c r}-P_{1 r}\right)\left(P_{c r}-P_{2 r}\right)} e^{\frac{N_{0}}{P_{c r}}} E_{1}\left(\frac{N_{0}}{P_{c r}}\right)
\end{aligned}
$$

and

$$
\begin{aligned}
R_{\text {mac }}^{\text {Sym }}= & \frac{P_{d r}^{2}+N_{0} P_{c r}-N_{0} P_{d r}-2 P_{d r} P_{c r}}{\left(P_{c r}-P_{d r}\right)^{2}} e^{\frac{N_{0}}{P_{d r}}} E_{1}\left(\frac{N_{0}}{P_{d r}}\right) \\
& +\frac{P_{d r}\left(2 P_{c r}-P_{d r}\right)}{\left(P_{c r}-P_{d r}\right)^{2}} e^{\frac{N_{0}}{P_{c r}}} E_{1}\left(\frac{N_{0}}{P_{c r}}\right)-\frac{P_{d r}}{P_{c r}-P_{d r}} .
\end{aligned}
$$

Note that the the exponential integral of first order is defined as

$$
E_{1}(z)=\int_{z}^{\infty} \frac{e^{-t}}{t} d t
$$

Proof See Appendix 1.

According to Theorem 1 in [41], we find that the function

$$
h(x) \triangleq \exp \left(\frac{1}{x}\right) E_{1}\left(\frac{1}{x}\right)
$$

is a monotonically increasing function with $x$. Based on this result, if $a>b$, it leads to

$$
e^{\frac{N_{0}}{a}} E_{1}\left(\frac{N_{0}}{a}\right)>e^{\frac{N_{0}}{b}} E_{1}\left(\frac{N_{0}}{b}\right) \text {. }
$$

Then we have

$$
\frac{a}{a-b}\left[e^{\frac{N_{0}}{a}} E_{1}\left(\frac{N_{0}}{a}\right)-e^{\frac{N_{0}}{b}} E_{1}\left(\frac{N_{0}}{b}\right)\right]>0 .
$$

The same result can be obtained for the case $a<b$. That is, the values of the expressions in (16)-(19) are always positive.

Our result in Theorem 1, in contrast, presents the exact closed-form expression which is applicable for arbitrary system parameters, and is given in closed-form expressions involving standard functions which can be easily evaluated using Matlab or Mathematics softwares. We note that this theorem presents an exact expression for the ergodic rate of the D2D communication aided by a DF relay node. In prior works, separate alternative expressions were only obtained for the traditional D2D communication scenarios without considering the different interference level in different time slots. Moreover, based on Theorem 1, we have the following observations. Since $e^{1 / x} E_{1}(1 / x)$ is a monotonically increasing function, Theorem 1 implies that $P_{1 r}>P_{c r}, \beta P_{r 2}>P_{b 2}, P_{2 r}>P_{c r}$, 
and $(1-\beta) P_{r 1}>P_{b 1}$ should hold to transmit the message between different nodes reliably. These conditions also mean that the interferences from the BS and the cellular user play a negative role in the ergodic rate.

\subsubsection{Weak interference case}

In this subsection, we examine the scenario that the D2D communication occurs at the cell edge where the D2D users reuse the resources of the cellular user far away. Hence, the interference at D2D users is weak enough compared to the noise which means $P_{c r} \rightarrow 0, P_{b i} \rightarrow 0$, $N_{0} / P_{c r} \rightarrow \infty$ and $N_{0} / P_{b i} \rightarrow \infty$. According to these, the ergodic rate of the D2D link can be described in the following corollary.

Corollary 1 When $N_{0} / P_{c r} \rightarrow \infty$ and $N_{0} / P_{b i} \rightarrow \infty$, we have

$$
R_{\text {sum }}^{W I}=\frac{1}{2} \min \left(R_{\text {mac }}^{W I}, R_{1}^{W I}+R_{1}^{W I}\right)
$$

where

$$
\begin{aligned}
& R_{1}^{W I}=\min \left(R_{1 r}^{W I}, R_{r 2}^{W I}\right), \\
& R_{2}^{W I}=\min \left(R_{2 r}^{W I}, R_{r 1}^{W I}\right),
\end{aligned}
$$

with

$$
\begin{aligned}
R_{1 r}^{W I} & =e^{\frac{N_{0}}{P_{1 r}}} E_{1}\left(\frac{N_{0}}{P_{1 r}}\right), \\
R_{r 2}^{W I} & =e^{\frac{N_{0}}{\beta P_{r 2}}} E_{1}\left(\frac{N_{0}}{\beta P_{r 2}}\right), \\
R_{2 r}^{W I} & =e^{\frac{N_{0}}{P_{2 r}}} E_{1}\left(\frac{N_{0}}{P_{2 r}}\right), \\
R_{r 1}^{W I} & =e^{\frac{N_{0}}{(1-\beta) P_{r 1}}} E_{1}\left(\frac{N_{0}}{(1-\beta) P_{r 1}}\right)
\end{aligned}
$$

and

$$
R_{\text {mac }}^{W I}= \begin{cases}R_{\text {mac }}^{W I, A s y} & \text { for the asymmetric case, } \\ R_{\text {mac }}^{W I, S y} & \text { for the symmetric case, }\end{cases}
$$

with

$$
\begin{aligned}
R_{\text {mac }}^{W I, A s y}= & \frac{P_{1 r}}{P_{1 r}-P_{2 r}} e^{\frac{N_{0}}{P_{1 r}}} E_{1}\left(\frac{N_{0}}{P_{1 r}}\right) \\
& +\frac{P_{2 r}}{P_{2 r}-P_{1 r}} e^{\frac{N_{0}}{P_{2 r}}} E_{1}\left(\frac{N_{0}}{P_{2 r}}\right)
\end{aligned}
$$

and

$$
R_{m a c}^{W I, S y}=\frac{P_{d r}-N_{0}}{P_{d r}} e^{\frac{N_{0}}{P_{d r}}} E_{1}\left(\frac{N_{0}}{P_{d r}}\right)+1 .
$$

Proof Based on the properties of exponential integral function, we can get

$$
\frac{1}{2} \ln \left(1+\frac{2}{x}\right)<e^{x} E_{1}(x)<\ln \left(1+\frac{1}{x}\right) .
$$

For $x \rightarrow \infty$, we have

$$
\left.e^{x} E_{1}(x)\right|_{x \rightarrow \infty} \approx 0 .
$$

Having these results, we can easily obtain the approximations in Corollary 1.

Corollary 1 provides approximate results of ergodic rate for the weak interference scenario. Clearly, the expressions in Corollary 1 are simpler than the ergodic rate expressions given in Theorem 1. Note that the ergodic rate only depends on the desired signal for the weak interference scenario. Since $h(x)$ in (24) is a monotonically increasing function with $x, R_{\text {sum }}^{W I}$ can be improved by increasing $P_{i r}$ and $P_{r i}$. That is to say, enhancing the power of desired signals can improve the performance of D2D link when the locations of D2D users are fixed.

\subsubsection{High SNR case}

Here, we consider the fact that the communicating users in D2D communication systems are relatively close to each other. Here we will present new asymptotic ergodic rate expressions when the receive SNR at the D2D users goes to infinity which means $P_{i r} / N_{0} \rightarrow \infty$ and $P_{r i} / N_{0} \rightarrow$ $\infty$. This will be useful in deriving the optimal power allocation for the ergodic rate at high SNR later in this section. For this case, the ergodic rate of D2D communication aided by the relay node are given in the following corollary.

Corollary 2 When $P_{\text {ir }} / N_{0} \rightarrow \infty$ and $P_{r i} / N_{0} \rightarrow \infty$, the asymptotic ergodic rate is given by

$$
R_{\text {sum }}^{H S}=\frac{1}{2} \min \left(R_{\text {mac }}^{H S}, R_{1}^{H S}+R_{2}^{H S}\right)
$$

where

$$
\begin{aligned}
& R_{1}^{H S}=\min \left(R_{1 r}^{H S}, R_{r 2}^{H S}\right), \\
& R_{2}^{H S}=\min \left(R_{2 r}^{H S}, R_{r 1}^{H S}\right),
\end{aligned}
$$

with

$$
\begin{aligned}
& R_{1 r}^{H S}=\ln \left(\frac{P_{1 r}}{N_{0}}\right)-\lambda-e^{\frac{N_{0}}{P_{c r}}} E_{1}\left(\frac{N_{0}}{P_{c r}}\right), \\
& R_{r 2}^{H S}=\ln \left(\frac{\beta P_{r 2}}{N_{0}}\right)-\lambda-e^{\frac{N_{0}}{P_{b 2}}} E_{1}\left(\frac{N_{0}}{P_{b 2}}\right), \\
& R_{2 r}^{H S}=\ln \left(\frac{P_{2 r}}{N_{0}}\right)-\lambda-e^{\frac{N_{0}}{P_{c r}}} E_{1}\left(\frac{N_{0}}{P_{c r}}\right), \\
& R_{r 1}^{H S}=\ln \left(\frac{(1-\beta) P_{r 1}}{N_{0}}\right)-\lambda-e^{\frac{N_{0}}{P_{b 1}}} E_{1}\left(\frac{N_{0}}{P_{b 1}}\right),
\end{aligned}
$$

and

$$
R_{\text {mac }}^{H S}=\left\{\begin{array}{c}
R_{m \text { mac }}^{H S, A s y} \text { for the asymmetric case, } \\
R_{\text {mac }}^{H S, S y} \text { for the symmetric case, }
\end{array}\right.
$$


with

$$
\begin{aligned}
R_{m a c}^{H S, A s y}= & \frac{P_{2 r}^{2}\left(\ln \left(P_{2 r} / N_{0}\right)-\lambda\right)}{\left(P_{2 r}-P_{1 r}\right)\left(P_{2 r}-P_{c r}\right)}-\frac{P_{1 r}^{2}\left(\ln \left(P_{1 r} / N_{0}\right)-\lambda\right)}{\left(P_{2 r}-P_{1 r}\right)\left(P_{1 r}-P_{c r}\right)} \\
& -\frac{P_{1 r} P_{2 r}-P_{c r}\left(P_{1 r}+P_{2 r}\right)}{\left(P_{c r}-P_{1 r}\right)\left(P_{c r}-P_{2 r}\right)} e^{\frac{N_{0}}{P_{c r}}} E_{1}\left(\frac{N_{0}}{P_{c r}}\right)
\end{aligned}
$$

and

$$
\begin{aligned}
R_{m a c}^{H S, S y}= & \frac{2 P_{d r} P_{c r}-P_{d r}^{2}}{\left(P_{c r}-P_{d r}\right)^{2}} \times\left[e^{\frac{N_{0}}{P_{c r}}} E_{1}\left(\frac{N_{0}}{P_{c r}}\right)-\ln \left(\frac{P_{d r}}{N_{0}}\right)+\lambda\right] \\
& +\frac{N_{0}}{P_{c r}-P_{d r}}\left(\ln \left(\frac{P_{c r}}{N_{0}}\right)-\lambda\right)-\frac{P_{d r}}{P_{c r}-P_{d r}} .
\end{aligned}
$$

where $\lambda \approx 0.577$ is the Euler-Mascheroni constant.

Proof We use the following asymptotic expansion of the exponential integral in obtaining the limiting form as $\mathrm{SNR} \rightarrow \infty$,

$$
\left.E_{1}(x)\right|_{x \rightarrow 0} \approx \ln \left(\frac{1}{x}\right)-\lambda .
$$

For the case the relay has some statistical channel state information (CSI) about the system parameters, $\beta$ may be chosen such that the ergodic rate is maximized. In this paper, we investigate the power allocation scheme that maximizes the ergodic rate based on the network geometry and the statistical CSI which include the second-order statistics and the interference level. For simplicity, we consider a linear network topology and assume that the relay node has only the path-loss coefficients of all the channels. We assume the transmit power at each user is $P_{T}$. Since the D2D communication always occurs far from the BS and the distance between D2D users is short, we can get the approximation that $d_{b 1} \approx d_{b 2}$ which leads to $P_{b 1} \approx P_{b 2} \approx P_{b}$.

Corollary 3 The power allocation strategy that maximizes the ergodic rate (39) is given by

$$
\beta^{*}=\left\{\begin{array}{c}
A \text { if }\left(G_{1}<0, A<1\right) \text { or }\left(G_{1}>0,0.5<B \leq A<1, g_{2 r}>g_{1 r}\right) \\
\text { or }\left(G_{1}>0, B \leq A<0.5, g_{2 r}>g_{1 r}\right) \\
B \text { if }\left(G_{1}>0,0.5<B \leq A<1, g_{1 r}>g_{2 r}\right) \text { or }\left(G_{1}>0,0.5<B<1 \leq A\right) \\
\quad \text { or }\left(G_{1}>0, B \leq A<0.5, g_{1 r}>g_{2 r}\right) \\
1 \text { if }\left(G_{1}<0, A>1\right) \\
0.5 \text { if }\left(G_{1}>0, B \leq 0.5 \leq A\right)
\end{array}\right.
$$

where

$$
\begin{aligned}
& A=\frac{g_{1 r} e^{\theta}}{g_{2 r}}, \\
& B=1-\frac{g_{2 r} e^{\theta}}{g_{1 r}},
\end{aligned}
$$

and

$$
\begin{gathered}
G_{1}=\left(g_{1 r}^{2}+g_{2 r}^{2}\right) e^{\theta}-g_{1 r} g_{2 r}, \\
\text { with } g_{s t}=d_{s t}^{-\alpha}(s, t=1,2, r ; s \neq t) .
\end{gathered}
$$

Proof See Appendix 2.

From the power allocation scheme in (50), we find that the power allocation coefficient is determined by the location of the relay node on the D2D link when the interference from cellular link is fixed. Instead, the interference level of the cellular link leads to difference power allocation coefficient for the fixed location of relay node. Note that the power allocation strategy in (50) uses only the second-order statistics and the interference levels from the cellular link. It means that the derived power allocation strategy can be easily implemented in practice. It is interesting that there exists some region for the optimal coefficient which equals to 0.5 .

\section{Numerical results}

In this section, we present several numerical results to demonstrate all the exact and analytical results as well as asymptotic results. The performance enhancements by the power allocation schemes are also shown here. The path-loss fading coefficient is considered in an urban macro cell. We set the reference distance to be unity. The simulation parameters are shown in Table 1.

Figure 3 illustrates the ergodic rate results. In this figure, we consider that the $\mathrm{D} 2 \mathrm{D}$ communication takes place in the middle region of the cell and is not far away from the cellular user with $d_{c r}=4, d_{b 1}=5$, and $d_{b 2}=6$. For the asymmetric case, we set $d_{1 r}=0.3, d_{2 r}=0.7$ and $\beta=0.6$ while $d_{1 r}=d_{2 r}=0.5$ and $\beta=0.5$ for the symmetric case. Again, we see a precise agreement between the analytical results and the Monte-Carlo simulations, and that the analytical approximations are accurate in the high SNR regime. Compared with the asymmetric case, we see that increasing the SNR over the threshold results in a small ergodic rate for the symmetric case. This agrees with the analytic conclusions given in Section 3 . It is also interesting to observe that the opposite occurs in the low SNR regime (and an improvement in system performance). In addition, it can be seen that there is a turning point in

Table 1 Simulation parameters

\begin{tabular}{ll}
\hline Transmit power of each user & $P_{i T}=P_{T}(i=C, 1,2, R)$ \\
Transmit power of BS & $P_{b T}=20 P_{i T}$ \\
Path-loss coefficients & $\alpha=4$ \\
Distance between D2D users & $d_{12}=1$ \\
Noise variance & $N_{0}=1$ \\
SNR & SNR $=P_{T} / N_{0}$ \\
\hline
\end{tabular}




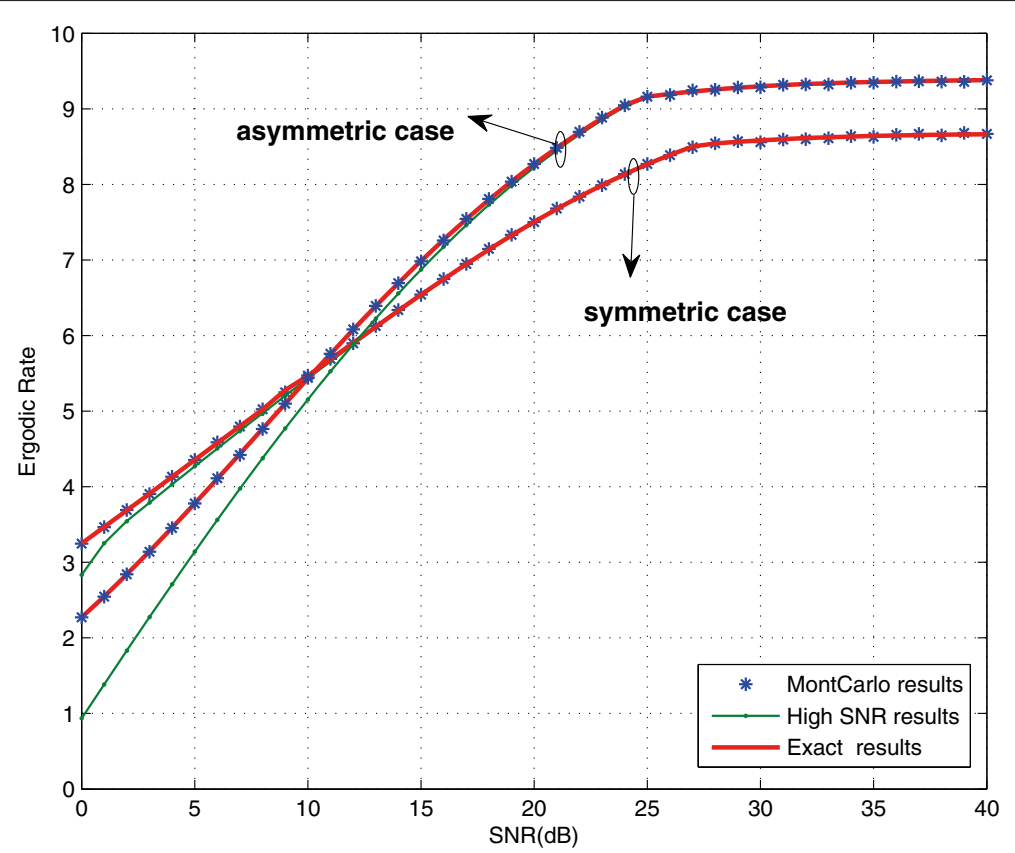

Fig. 3 Exact ergodic rate, high SNR approximations, and Monte-Carlo simulated ergodic rate for both asymmetric and symmetric cases of D2D communication assisted by two-way DF relay node

all the curves for both cases. This is explained as follows. Before this point, due to the minimization operation in (15), the ergodic rate is given by one of $R_{\text {mac }}$ or $R_{1}+R_{2}$ which completely depends on the interference level of the BS and the cellular user. After this point, the other one determines the ergodic rate due to the large SNR.
We now assess the approximations of ergodic rate for the weak interference condition in Figs. 4 and 5. Considering the D2D communication occurring at the edge of the cell, in Fig. 4, we set $d_{b 1}=d_{b 2}=10$ and $d_{c r}=8$ for the symmetric case while $d_{1 r}=0.4, d_{2 r}=0.6$, and $d_{b 1}=d_{b 2}=d_{c r}=14$ are set for the asymmetric case.

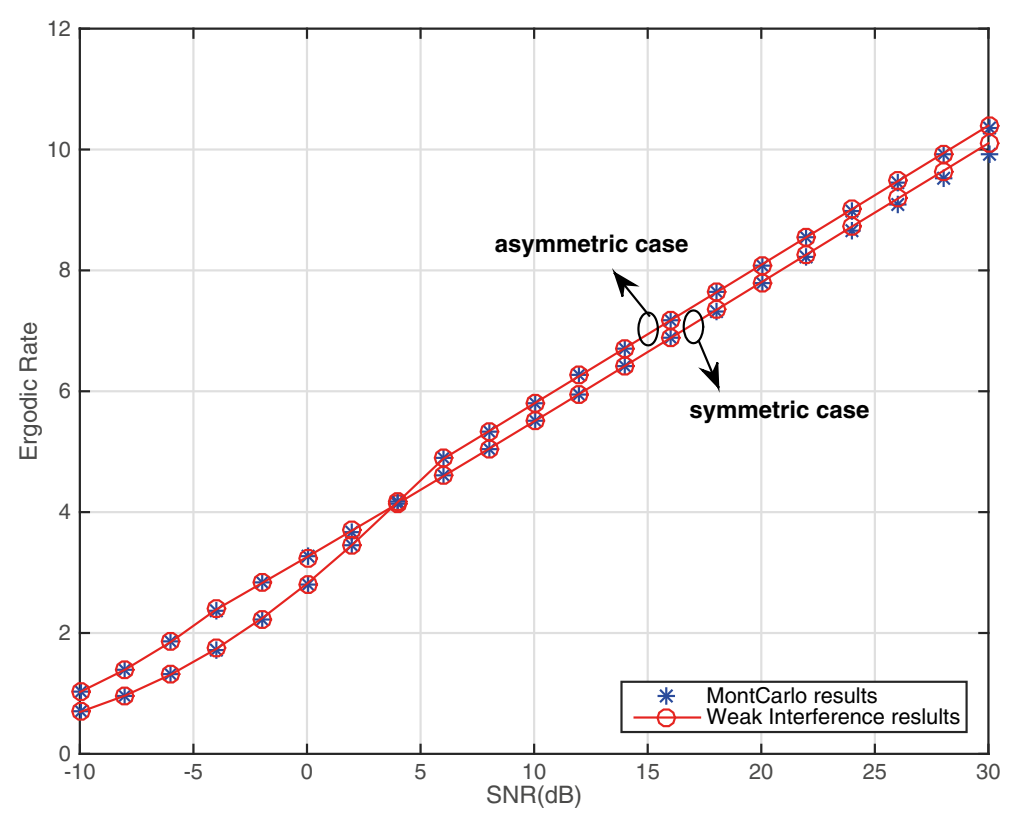

Fig. 4 Weak interference approximations and Monte-Carlo simulated ergodic rate against SNR for both asymmetric and symmetric cases 


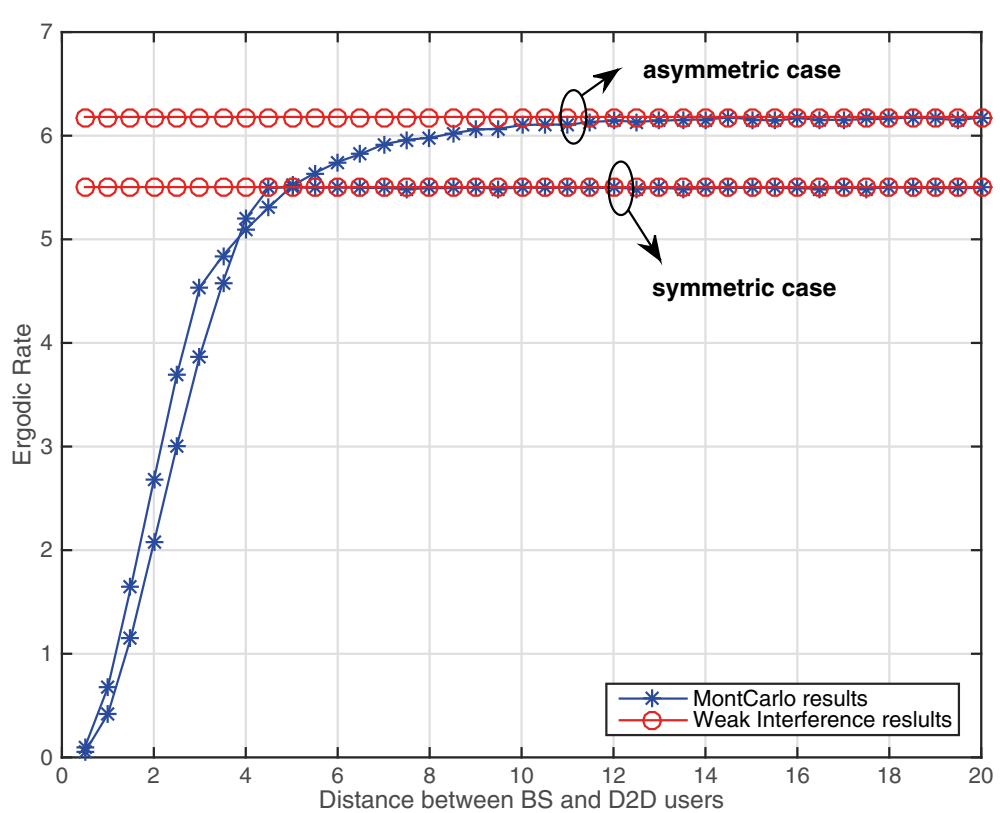

Fig. 5 Weak interference approximations and Monte-Carlo simulated ergodic rate against the distance between BS and D2D users for both asymmetric and symmetric cases

We also assume $d_{c r}=6$ and SNR $=10 \mathrm{~dB}$ for both symmetric and asymmetric cases in Fig. 5. Specially, in Fig. 5, we set $d_{1 r}=0.3$ and $d_{2 r}=0.7$ for the asymmetric case. It is apparent that the weak interference asymptotic expressions almost coincide with the Monte-Carlo results in the whole SNR regime. The graph in Fig. 5 shows that the approximations in Corollary 1 remain sufficiently tight in the weak interference regime. As anticipated, the distance between BS and D2D users ceiling effect kicks in at impractical distance values (e.g., > 10).

Figure 6 shows the ergodic rate results of the systems in different parameter configurations, where the parameters
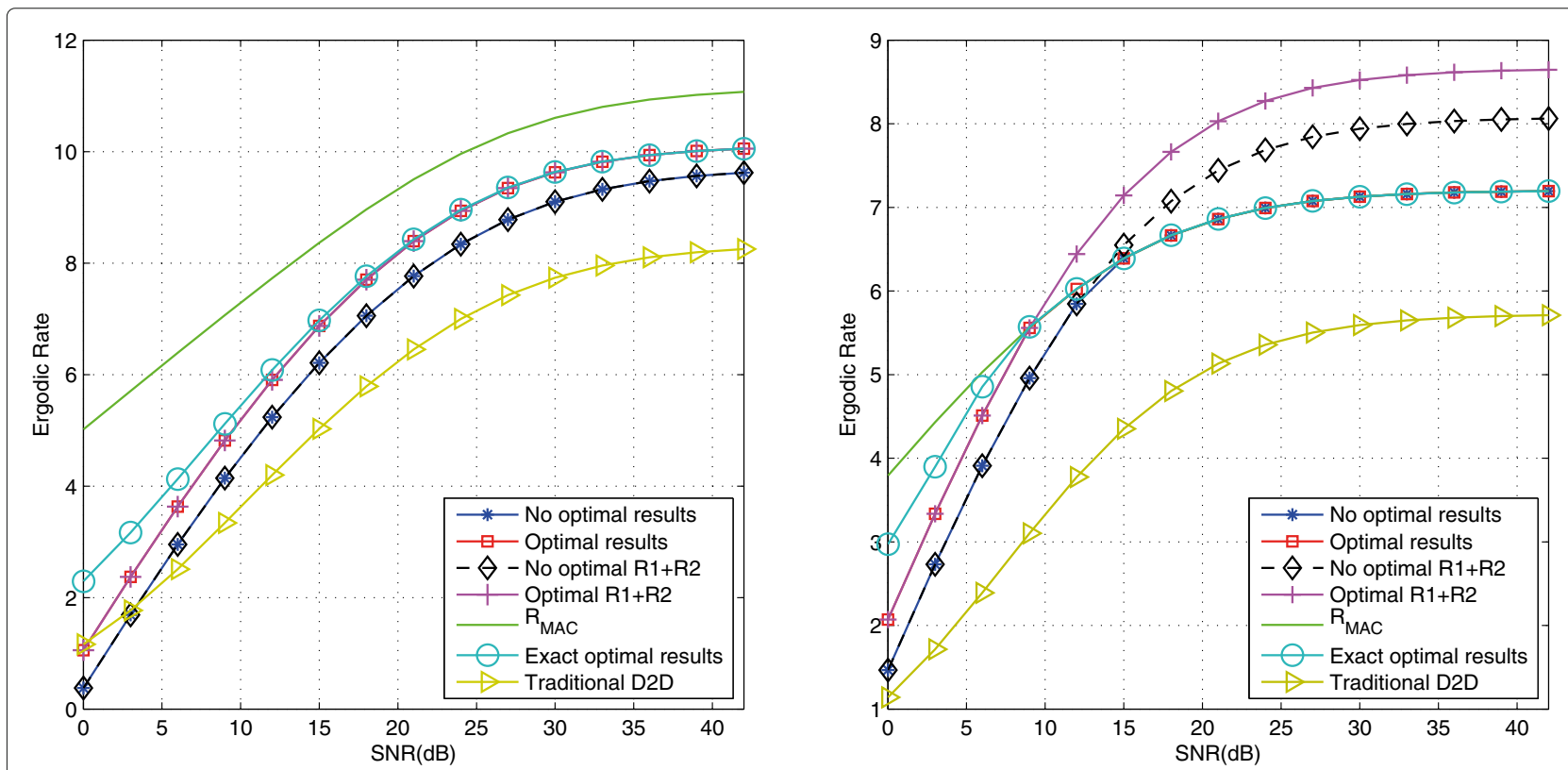

Fig. 6 Ergodic rate performance comparison of the proposed power allocation strategy against the SNR for different parameters of $\left(d_{c r}, d_{1 r}, d_{2 r}\right)=(4,0.25,0.75)$ and $\left(d_{c r}, d_{1 r}, d_{2 r}\right)=(2,0.35,0.65)$. The equal power allocation and traditional D2D communication results are presented for comparison 
of the left one are $\left(d_{c r}, d_{1 r}, d_{2 r}\right)=(4,0.25,0.75)$ while those for the right one are $\left(d_{c r}, d_{1 r}, d_{2 r}\right)=(2,0.35,0.65)$. The results for the traditional D2D communication are also presented for comparison. The star line in the left figure represents the ergodic rate of the scenario without power allocation while the square red line demonstrates the result utilizing power allocation strategy. As we employ the power allocation scheme in the broadcast phase, the ergodic rate in this phase is improved compared with non-optimized black link. Since the DF protocol is employed in the relay node, the ergodic rate of the whole system is determined by the minimum rate during the broadcast phase and the multiple-access channel (MAC) phase as in Theorem 1. The ergodic rate in square red line performs better than that in star blue line for all SNRs. Similarly, in the right figure, the ergodic rate of the nonoptimized system equals to that of MAC phase if the SNR is no more than $13 \mathrm{~dB}$ while it depends on the ergodic rate in the MAC phase if the SNR is above $13 \mathrm{~dB}$. Using the power allocation strategy, the ergodic rate is the same as that in the MAC phase resulting in the distinct performance improvements when the SNR is below $13 \mathrm{~dB}$. The exact optimal results in this figure test the availability of our power allocation strategy.

Finally, Fig. 7 compares the ergodic rates of the relayassisted D2D communication strategy with and without the power allocation strategy against different relay node locations. We consider $\left(d_{b 1}, d_{b 2}, d_{c r}\right)=(5,5,5), \beta=0.5$ for the left subfigure and $\left(d_{b 1}, d_{b 2}, d_{c r}\right)=(4,4,4), \beta=0.3$ for the right one. As can be seen in the left subfigure, again, the proposed power allocation algorithm (50) outperforms the equal power allocation significantly. It can be easily found that the ergodic rate depends on the location of the relay node. It is also shown that more ergodic rate gain can be obtained comparing to the equal power allocation when the relay node is closer to the D2D users. This phenomena highlights the fact that the power allocation strategy is particularly effective when the distances between D2D users and relay node are different. From the right subfigure, since $\beta$ is set as 0.3 , the ergodic rate in the region $d_{1 r}>0.7\left(d_{2 r}<0.3\right)$ is close to the optimal results. It means the closer the relay node to the D2D user, the less power should be allocated. Again, an agreement between exact results and high SNR optimal results are shown in the figure.

\section{Conclusions}

This paper proposed a new D2D communication strategy underlaying cellular networks which is aided by a relay node using two-way DF protocol. The outage probability and the ergodic rate of the new strategy were discussed for both the asymmetric and symmetric cases. For the new D2D communication strategy, we derive the closedform expressions and their high SNR approximations for both outage probability and the ergodic rate. Based on these results, we showed that several major factors play a negative role for the system performance. Furthermore, closed-form power allocation solutions which minimize
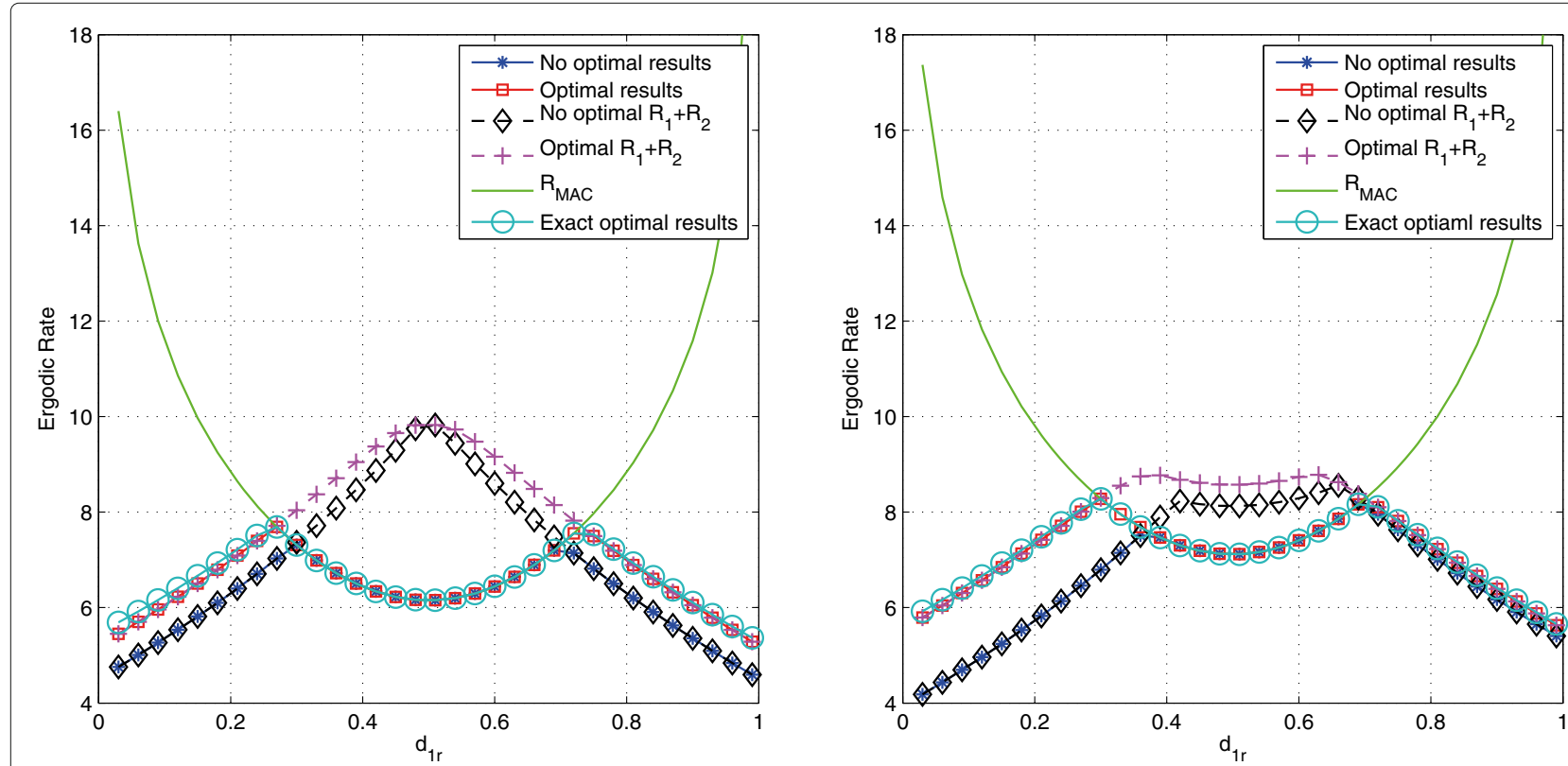

Fig. 7 Ergodic rate performance comparison of the proposed power allocation strategy against different relay node locations and SNRs, including $\left(d_{b 1}, d_{b 2}, d_{c r}\right)=(5,5,5), \beta=0.5$ for the left subfigure and $\left(d_{b 1}, d_{b 2}, d_{c r}\right)=(4,4,3), \beta=0.3$ for the right one. The exact optimal results are presented for comparison 
the outage probability or maximizes the ergodic rate were presented using the high SNR approximations. Analytical results were validated through numerical simulations.

\section{Appendices}

\section{Appendix 1}

\section{Proof of Theorem 1}

In order to prove Theorem 1, we will consider the asymmetric and symmetric cases, respectively. Note that, in both cases, we use the following equation for the evaluation of the exact ergodic rate [42]

$$
\begin{aligned}
E_{X}[\ln (1+x)] & =\int_{0}^{\infty} \ln (1+x) d F(x) \\
& =\int_{0}^{\infty} \frac{1-F_{X}(x)}{x+1} d x
\end{aligned}
$$

Here, we start from the asymmetric case.

(1) The Asymmetric Case

Without loss of generality, let $S=P_{1 r}\left|h_{1 r}\right|^{2}+$ $P_{2 r}\left|h_{2 r}\right|^{2}, T=P_{c r}\left|h_{c r}\right|^{2}+N_{0}$ and $U=S / T$. First, we derive the PDF of $S$. Let $X=P_{1 r}\left|h_{1 r}\right|^{2}, Y=P_{2 r}\left|h_{2 r}\right|^{2}$ and $S=X+Y$. For the Rayleigh fading channel, we have

$$
f(x)=\frac{1}{P_{1 r}} e^{-\frac{x}{P_{1 r}}}
$$

and

$$
f(y)=\frac{1}{P_{2 r}} e^{-\frac{y}{P_{2 r}}}
$$

Then, we have

$$
f_{S}(s) d s=\int_{-\infty}^{\infty} f(s-y, y) d y d s
$$

Since random variables $X, Y$ are independent and $X>0, Y>0$, we have

$$
\begin{aligned}
f(s) & =\int_{0}^{s} f_{X}(s-y) f_{y}(y) d y \\
& =\frac{1}{P_{1 r} P_{2 r}} e^{-\frac{s}{P_{1 r}}} \int_{0}^{s} e^{-\left(\frac{1}{P_{2 r}}-\frac{1}{P_{1 r}}\right) y} d y \\
& =\frac{1}{P_{2 r}-P_{1 r}} e^{-\frac{s}{P_{2 r}}}-\frac{1}{P_{2 r}-P_{1 r}} e^{-\frac{s}{P_{1 r}}}
\end{aligned}
$$

The same way, we can easily derive the PDF of $T$ as

$$
f_{T}(t)=\frac{1}{P_{c r}} e^{-\frac{t}{P_{c r}}+\frac{N_{0}}{P_{c r}}} .
$$

Then we evaluate the PDF of $U$ as

$$
\begin{aligned}
f_{U}^{a s y}(u)= & \int_{N_{0}}^{\infty} t f(u t, t) d t \\
= & \int_{N_{0}}^{\infty} t f_{S}(u t) f_{T}(t) d t \\
= & \frac{e^{\frac{N_{0}}{P_{c r}}}}{P_{c r}\left(P_{2 r}-P_{1 r}\right)} \frac{P_{2 r}}{u+\frac{P_{2 r}}{P_{c r}}} N_{0} e^{-\frac{u}{P_{2 r}}-\frac{N_{0}}{P_{c r}}} \\
& +\frac{e^{\frac{N_{0}}{P_{c r}}}}{P_{c r}\left(P_{2 r}-P_{1 r}\right)}\left(\frac{P_{2 r}}{u+\frac{P_{2 r}}{P_{c r}}}\right)^{2} e^{-\frac{u}{P_{2 r}}-\frac{N_{0}}{P_{c r}}} \\
& -\frac{e^{\frac{N_{0}}{P_{c r}}}}{P_{c r}\left(P_{2 r}-P_{1 r}\right)} \frac{P_{1 r}}{u+\frac{P_{1 r}}{P_{c r}}} N_{0} e^{-\frac{u}{P_{1 r}}-\frac{N_{0}}{P_{c r}}} \\
& -\frac{e^{\frac{N_{0}}{P_{c r}}}}{P_{c r}\left(P_{2 r}-P_{1 r}\right)}\left(\frac{P_{1 r}}{u+\frac{P_{1 r}}{P_{c r}}}\right)^{2} e^{-\frac{u}{P_{1 r}}-\frac{N_{0}}{P_{c r}}}
\end{aligned}
$$

where $f(u t, t)$ is the joint PDF of the random variables $S$ and $T$. After simple manipulation, it yields

$$
\begin{aligned}
f_{U}^{a s y}(u)= & \frac{P_{2 r} N_{0}}{P_{c r}\left(P_{2 r}-P_{1 r}\right)}\left(\frac{1}{u+\frac{P_{2 r}}{P_{c r}}}\right) e^{-\frac{N_{0}}{P_{2 r}} u} \\
& +\frac{P_{2 r}^{2}}{P_{c r}\left(P_{2 r}-P_{1 r}\right)}\left(\frac{1}{u+\frac{P_{2 r}}{P_{c r}}}\right)^{2} e^{-\frac{N_{0}}{P_{2 r}} u} \\
& -\frac{P_{1 r} N_{0}}{P_{c r}\left(P_{2 r}-P_{1 r}\right)}\left(\frac{1}{u+\frac{P_{1 r}}{P_{c r}}}\right) e^{-\frac{N_{0}}{P_{1 r}} u} \\
& -\frac{P_{1 r}^{2}}{P_{c r}\left(P_{2 r}-P_{1 r}\right)}\left(\frac{1}{u+\frac{P_{1 r}}{P_{c r}}}\right)^{2} e^{-\frac{N_{0}}{P_{1 r}} u} .
\end{aligned}
$$

Then we integrate (65) from $\gamma_{\text {thm }}$ to $\infty$ to yield

$$
\begin{aligned}
F_{\text {mac }}^{A s y}= & \frac{P_{1 r}^{2}}{\left(P_{2 r}-P_{1 r}\right)\left(P_{c r} \gamma_{t h m}+P_{1 r}\right)} e^{-\frac{N_{0}}{P_{1 r}} \gamma_{t h m}} \\
& -\frac{P_{2 r}^{2}}{\left(P_{2 r}-P_{1 r}\right)\left(P_{c r} \gamma_{t h m}+P_{2 r}\right)} e^{-\frac{N_{0}}{P_{2 r}} \gamma_{t h m}} .
\end{aligned}
$$

Then, we can derive the exact expression for $R_{m a c}$ as

$$
\begin{aligned}
R_{\text {mac }}^{A s y}= & \underbrace{\frac{-P_{1 r}^{2}}{P_{2 r}-P_{1 r}} \int_{0}^{\infty} \frac{e^{-\frac{N_{0}}{P_{1 r}} x}}{\left(P_{c r} x+P_{1 r}\right)(x+1)} d x}_{I_{3}} \\
& +\underbrace{\frac{-P_{2 r}^{2}}{P_{2 r}-P_{1 r}} \int_{0}^{\infty} \frac{e^{-\frac{N_{0}}{P_{2 r}} x}}{\left(P_{c r} x+P_{2 r}\right)(x+1)} d x}_{I_{4}} .
\end{aligned}
$$


It can be seen that $I_{3}$ and $I_{4}$ have a similar structure. Hence, we only focus on $I_{3}$ in this proof. Then $I_{3}$ can be reexpressed as

$I_{3}=\frac{-P_{1 r}^{2}}{P_{c r}\left(P_{2 r}-P_{1 r}\right)} \int_{0}^{\infty}\left(\frac{1}{\left(x+\frac{P_{1 r}}{P_{c r}}\right)}-\frac{1}{x+1}\right)\left(\frac{1}{1-\frac{P_{1 r}}{P_{c r}}}\right) e^{-\frac{N_{0}}{P_{1 r}} x} d x$

Applying the following identity [43]:

$$
\int_{0}^{\infty} \frac{e^{-u x} d x}{x+\beta}=-e^{\beta u} E_{i}(-u \beta),
$$

we have

$$
I_{3}=\frac{P_{1 r}^{2}}{\left(P_{2 r}-P_{1 r}\right)\left(P_{c r}-P_{1 r}\right)}\left[e^{\frac{N_{0}}{P_{c r}}} E_{i}\left(-\frac{N_{0}}{P_{c r}}\right)-e^{\frac{N_{0}}{P_{1 r}}} E_{i}\left(-\frac{N_{0}}{P_{1 r}}\right)\right] .
$$

Similarly, $I_{4}$ can be obtained as

$$
I_{4}=\frac{P_{2 r}^{2}}{\left(P_{2 r}-P_{1 r}\right)\left(P_{c r}-P_{2 r}\right)}\left[e^{\frac{N_{0}}{P_{2 r}}} E_{i}\left(-\frac{N_{0}}{P_{2 r}}\right)-e^{\frac{N_{0}}{P_{c r}}} E_{i}\left(-\frac{N_{0}}{P_{c r}}\right)\right] .
$$

We can also get (16)-(19) by using the same approach. Utilizing the recursive relation

$$
E_{1}(z)=-E i(-z)
$$

and then combining (67) yields the desired result.

\section{(2) The Symmetric Case}

Let $S=P_{d r}\left|h_{1 r}\right|^{2}+P_{d r}\left|h_{2 r}\right|^{2}, T=P_{c r}\left|h_{c r}\right|^{2}+N_{0}$ and $U=S / T$. We can easily get the PDFs of $S$ and $T$ as

$$
f_{S}(s)=\left(1 / P_{d r}\right)^{2} s e^{-s / P_{d r}}
$$

and

$$
f_{T}(t)=1 / P_{c r} e^{-t / P_{c r}+N_{0} / P_{c r}} .
$$

Then the PDF of $U$ can be obtained as

$$
\begin{aligned}
f_{U}^{s y}(u)= & \frac{u}{P_{d r}^{2} P_{c r}} e^{\frac{N_{0}}{P_{c r}}} \int_{N_{0}}^{\infty} t^{2} e^{\left(-\frac{u}{P_{d r}}-\frac{1}{P_{c r}}\right) t} d t \\
= & \frac{2 P_{d r}}{P_{c r}} \frac{u e^{-\frac{N_{0}}{P_{d r}} u}}{\left(u+\frac{P_{d r}}{P_{c r}}\right)^{3}}+\frac{2 n}{P_{c r}} \frac{u e^{-\frac{N_{0}}{P_{d r}} u}}{\left(u+\frac{P_{d r}}{P_{c r}}\right)^{2}} \\
& +\frac{n^{2}}{P_{c r} P_{d r}} \frac{u e^{-\frac{N_{0}}{P_{d r}}} u}{u+\frac{P_{d r}}{P_{c r}}} .
\end{aligned}
$$

To proceed further, some transformations are performed

$$
\begin{aligned}
F_{\gamma_{\text {mac }}}^{S y}= & \frac{2 P_{d r}}{P_{c r}} \int_{\gamma_{t h}}^{\infty} \frac{u+\frac{P_{d r}}{P_{c r}}-\frac{P_{d r}}{P_{c r}}}{\left(u+\frac{P_{d r}}{P_{c r}}\right)^{3}} e^{-\frac{N_{0}}{P_{d r}} u} d u \\
& +\frac{2 n}{P_{c r}} \int_{\gamma_{t h}}^{\infty} \frac{u+\frac{P_{d r}}{P_{c r}}-\frac{P_{d r}}{P_{c r}}}{\left(u+\frac{P_{d r}}{P_{c r}}\right)^{2}} e^{-\frac{N_{0}}{P_{d r}} u} d u \\
& +\frac{N_{0}^{2}}{P_{c r} P_{d r}} \int_{\gamma_{t h}}^{\infty} \frac{u+\frac{P_{d r}}{P_{c r}}-\frac{P_{d r}}{P_{c r}}}{u+\frac{P_{d r}}{P_{c r}}} e^{-\frac{N_{0}}{P_{d r}} u} d u
\end{aligned}
$$

Then we evaluate the CDF of $U$ from $\gamma_{t h m}$ to $\infty$. With the help of $[43,(3.353)]$, the CDF can be derived as

$$
\begin{aligned}
F_{\text {mac }}^{S y}= & \left(\frac{2 P_{d r}}{P_{c r}}-\frac{2 N_{0} P_{d r}}{P_{c r}^{2}}\right) \\
& \times\left(\frac{e^{-\frac{N_{0}}{P_{d r}}} \gamma_{t h}}{\gamma_{t h}+\frac{P_{d r}}{P_{c r}}}+\frac{N_{0}}{P_{d r}} e^{\frac{N_{0}}{P_{c r}}} E i\left(-\frac{N_{0}}{P_{d r}} \gamma_{t h m}-\frac{N_{0}}{P_{c r}}\right)\right) \\
& +\left(\frac{2 N_{0}}{P_{c r}}-\frac{N_{0}^{2}}{P_{c r}^{2}}\right) \times\left(-e^{\frac{N_{0}}{P_{c r}}} E i\left(-\frac{N_{0}}{P_{d r}} \gamma_{t h}-\frac{N_{0}}{P_{c r}}\right)\right)+\frac{N_{0}}{P_{c r}} e^{-\frac{N_{0}}{P_{d r}} \gamma_{t h}} \\
& -\frac{2 P_{d r}^{2}}{P_{c r}^{2}} \times\left\{e^{-\frac{N_{0}}{P_{d r}} \gamma_{t h}}\left(-\frac{N_{0}}{2 P_{d r}\left(\gamma_{t h}+\frac{P_{d r}}{P_{c r}}\right)}+\frac{1}{2\left(\gamma_{t h}+\frac{P_{d r}}{P_{c r}}\right)^{2}}\right)\right. \\
& \left.-\frac{N_{0}^{2}}{2 P_{d r}^{2}} e^{\frac{N_{0}}{P_{c r}}} E i\left(-\frac{N_{0}}{P_{d r}} \gamma_{t h}-\frac{N_{0}}{P_{c r}}\right)\right\} .
\end{aligned}
$$

Based on the CDF in (77), we can evaluate the ergodic rate for $R_{\text {mac }}$ as

$$
\begin{aligned}
R_{m a c}^{S y}= & \underbrace{\int_{0}^{r} \frac{1}{y+\frac{P_{d r}}{P_{c r}}} e^{-\frac{N_{0}}{P_{d r}} y} \frac{1}{1+y} d y\left(\frac{2}{P_{r}}-\frac{N_{0}}{P_{c r}^{2}}\right) P_{d r}}_{I_{5}} \\
& +\underbrace{\int_{0}^{\infty} \frac{e^{-\frac{N_{0}}{P_{d r}} y}}{1+y} d y \frac{N_{0}}{P_{c r}}}_{I_{6}} \\
& -\underbrace{\int_{0}^{\infty} \frac{1}{\left(y+\frac{P_{d r}}{P_{c r}}\right)^{2}} \frac{1}{1+y} e^{-\frac{N_{0}}{P_{d r}} y} d y \frac{P_{d r}^{2}}{P_{c r}^{2}}}_{I_{7}} .
\end{aligned}
$$

Note that $I_{5}$ and $I_{6}$ can be derived by using the same method for the derivation of $I_{3}$. The exact expressions of $I_{5}$ and $I_{6}$ are then, respectively, given by

$$
I_{5}=\frac{P_{d r}\left(2 P_{c r}-N_{0}\right)}{P_{c r}\left(P_{c r}-P_{d r}\right)}\left[e^{\frac{N_{0}}{P_{d r}}} \operatorname{Ei}\left(-\frac{N_{0}}{P_{d r}}\right)-e^{\frac{N_{0}}{P^{d r}}} \operatorname{Ei}\left(-\frac{N_{0}}{P_{c r}}\right)\right]
$$


and

$$
I_{6}=\frac{-N_{0}}{P_{c r}} e^{\frac{N_{0}}{P_{d r}}} \operatorname{Ei}\left(-\frac{N_{0}}{P_{d r}}\right) .
$$

To evaluate the integral in $I_{7}$, we note that $I_{7}$ can be expressed by using (27) in [42] as

$$
I_{7}=\frac{P_{d r}^{2}}{P_{c r}^{2}} J_{1}\left(\frac{N_{0}}{P_{d r}}, \frac{P_{d r}}{P_{c r}}, 0,2\right),
$$

where

$$
J_{1}(a, b, m, n)=\int_{0}^{\infty} \frac{x^{m} e^{-a x}}{(x+b)^{n}(x+1)} d x .
$$

Utilizing the recursive relation in (27) of [42], $I_{7}$ can be expressed as

$$
\begin{aligned}
I_{7}= & \frac{P_{d r}^{2}}{\left(P_{c r}-P_{d r}\right) P_{c r}} J_{2}\left(\frac{N_{0}}{P_{d r}}, \frac{P_{d r}}{P_{c r}}, 0,2\right) \\
& +\frac{P_{d r}^{2}}{\left(P_{c r}-P_{d r}\right)^{2}} J_{2}\left(\frac{N_{0}}{P_{d r}}, 1,0,1\right) \\
& -\frac{P_{d r}^{2}}{\left(P_{c r}-P_{d r}\right)^{2}} J_{2}\left(\frac{N_{0}}{P_{d r}}, \frac{P_{d r}}{P_{c r}}, 0,1\right) .
\end{aligned}
$$

After some subsequent manipulations, we have

$J_{2}\left(\frac{N_{0}}{P_{d r}}, \frac{P_{d r}}{P_{c r}}, 0,2\right)=e^{\frac{N_{0}}{P_{c r}}}\left[\frac{P_{c r}}{P_{d r}} e^{-\frac{N_{0}}{P_{c r}}}-\frac{N_{0}}{P_{d r}} E_{1}\left(\frac{N_{0}}{P_{c r}}\right)\right]$,

$$
J_{2}\left(\frac{N_{0}}{P_{d r}}, 1,0,1\right)=e^{\frac{N_{0}}{P_{d r}}} J_{3}\left(\frac{N_{0}}{P_{d r}}, 1,-1\right)=e^{\frac{N_{0}}{P_{d r}}} \cdot E_{1}\left(\frac{N_{0}}{P_{d r}}\right),
$$

$$
J_{2}\left(\frac{N_{0}}{P_{d r}}, \frac{P_{d r}}{P_{c r}}, 0,1\right)=e^{\frac{N_{0}}{P_{c r}}} J_{3}\left(\frac{N_{0}}{P_{d r}}, \frac{P_{d r}}{P_{c r}},-1\right)=e^{\frac{N_{0}}{P_{c r}}} \cdot E_{1}\left(\frac{N_{0}}{P_{c r}}\right) .
$$

Substituting (84)-(86) into (83) yields

$$
\begin{aligned}
I_{7}= & \frac{P_{d r}^{2}}{\left(P_{c r}-P_{d r}\right) P_{c r}} \cdot e^{\frac{N_{0}}{P_{c r}}}\left[\frac{P_{c r}}{P_{d r}} e^{-\frac{N_{0}}{P_{c r}}}-\frac{N_{0}}{P_{d r}} E_{1}\left(\frac{N_{0}}{P_{c r}}\right)\right] \\
& +\frac{P_{d r}^{2}}{\left(P_{c r}-P_{d r}\right)^{2}}\left[e^{\frac{N_{0}}{P_{d r}}} E_{1}\left(\frac{N_{0}}{P_{d r}}\right)-e^{\frac{N_{0}}{P_{c r}}} E_{1}\left(\frac{N_{0}}{P_{c r}}\right)\right] .
\end{aligned}
$$

Substituting (79), (80) and (87) into (78), we get (22). Then utilizing (16)-(19) and (22) yields the desired result.

\section{Appendix 2}

\section{Proof of Corollary 3}

Note that $R_{\text {mac }}^{H S}$ in (39) is independent of $\beta$ and therefore, we consider the following maximization instead

$$
\beta^{*}=\arg \max _{\beta}\left[R_{1}^{H S}+R_{2}^{H S}\right] .
$$

We assume the transmit power at each user is $P_{T}$. From (42) to (45), we can get the following equation by assuming

$$
\begin{cases}A \geq B & G_{1}>0 \\ A \leq B & G_{1}<0\end{cases}
$$

where

$$
\begin{aligned}
& \theta_{1}=e^{\frac{N_{0}}{P_{b 2}}} E_{1}\left(\frac{N_{0}}{P_{b 2}}\right)-e^{\frac{N_{0}}{P_{c r}}} E_{1}\left(\frac{N_{0}}{P_{c r}}\right), \\
& \theta_{2}=e^{\frac{N_{0}}{P_{b 1}}} E_{1}\left(\frac{N_{0}}{P_{b 1}}\right)-e^{\frac{N_{0}}{P_{c r}}} E_{1}\left(\frac{N_{0}}{P_{c r}}\right),
\end{aligned}
$$

Since $P_{b 1} \approx P_{b 2} \approx P_{b}$, we have $\theta_{1} \approx \theta_{2} \approx \theta$. In order to get the optimal $\beta$, we will consider two cases: $A \leq B$ and $B \leq A$.

(1) The $A \leq B$ Case $\left(G_{1} \leq 0\right)$

In this case, $\beta$ will fall into one of the three cases, (1) $\beta \leq A$, (2) $A \leq \beta \leq B$, and (3) $B \leq \beta$. We firstly consider the $\beta \leq A$ scenario. The problem in (88) is now reduced to

$$
\beta^{*}=\arg \max _{\beta}\left[R_{2 r}^{H S}+R_{r 2}^{H S}\right],
$$

where $R_{2 r}^{H S}$ is independent of $\beta$ and $R_{r 2}^{H S}$ is a strictly increasing function with respect to $\beta$. It follows that $\beta^{*}=A$ is the optimal value.

For the $A \leq \beta \leq B$ scenario, the problem in (88) can be simplified as

$$
\beta^{*}=\arg \max _{\beta}\left[R_{1 r}^{H S}+R_{2 r}^{H S}\right]
$$

which is independent of $\beta$ which implies that no optimization is needed for this interval. For this case, we have

$$
R_{\max }^{A<\beta<B}=\ln \left(\frac{P_{1 r} P_{2 r}}{N_{0}^{2}}\right)-2 e^{\frac{N_{0}}{P_{c r}}} E_{1}\left(\frac{N_{0}}{P_{c r}}\right)-2 \lambda .
$$

We now consider the $B \leq \beta$ scenario. It is easy to simplify the problem in (88) as

$$
\beta^{*}=\arg \max _{\beta}\left[R_{1 r}^{H S}+R_{r 1}^{H S}\right] .
$$

Note that $R_{r 1}^{H S}$ is independent of $\beta$ while $R_{1 r}^{H S}$ is a strictly decreasing function with respect to $\beta$. It therefore follows that $\beta^{*}=B$ is the optimal value. Having the above results, we now derive the optimal results among (92), (93) and (95). By substituting $\beta^{*}=A$ into (92), we have

$\max \left[R_{2 r}^{H S}+R_{r 2}^{H S}\right]=\ln \left(\frac{P_{1 r} P_{2 r}}{N_{0}^{2}}\right)-2 e^{\frac{N_{0}}{P_{c r}}} E_{1}\left(\frac{N_{0}}{P_{c r}}\right)-2 \lambda$. 
Similarly, when $B \leq \beta$, we have

$$
\max \left[R_{1 r}^{H S}+R_{r 1}^{H S}\right]=\ln \left(\frac{P_{1 r} P_{2 r}}{N_{0}^{2}}\right)-2 e^{\frac{N_{0}}{P_{c r}}} E_{1}\left(\frac{N_{0}}{P_{c r}}\right)-2 \lambda .
$$

According to the results in (94)-(97), we find the maximum values of these three cases are the same. Furthermore, we consider the possibility regions of $A$ and $B$ under case $A \geq B$. Based on the expression of $A$, we have $A>0$. Since both $\beta^{*}=A$ and $\beta^{*}=B$ can lead to the maximum rate, when $A<1$, the value of $\beta^{*}$ is taken as $\beta^{*}=A$. When $A>1$, since Eq. (100) is an increasing function with respect to $\beta, \beta^{*}=1$ will be the optimal results for the maximization problem in (96). In conclusion, we have

$$
\beta^{*}=\left\{\begin{array}{ccc}
A & \text { if } & \left(G_{1}<0, A<1\right) \\
1 & \text { if } \quad\left(G_{1}<0, A<1\right)
\end{array}\right.
$$

Then, we consider the another case.

2) The $B \leq A$ Case $\left(G_{1} \geq 0\right)$

In this case, $\beta$ will fall into one of the three cases, (1) $\beta \leq B$, (2) $B \leq \beta \leq A$, and (3) $A \leq \beta$. After operating the same procedure in Section (1), we can easily obtain the following results

When $\beta \leq B$, we have

$$
R_{\max }^{\beta \leq B}=R_{2 r}^{H S}+R_{r 2}^{H S}\left(\beta^{*}=B\right) .
$$

When $\beta \geq A$, it yields

$$
R_{\max }^{\beta \geq A}=R_{1 r}^{H S}+R_{r 1}^{H S}\left(\beta^{*}=A\right) .
$$

For the case $B<\beta<A$, we have

$$
R_{\max }^{B \leq \beta \leq A}=R_{r 1}^{H S}(\beta)+R_{r 2}^{H S}(\beta) .
$$

By taking the derivative of $\beta$, we have

$$
R_{\max }^{B \leq \beta \leq A^{\prime}}=\frac{1}{\beta}-\frac{1}{1-\beta} .
$$

Then the optimal $\beta$ for $R_{\max }^{B \leq \beta \leq A}$ is $\beta^{*}=1 / 2$.

Now we find the maximum result among $R_{\max }^{\beta \leq B}$, $R_{\max }^{B \leq \beta \leq A}$ and $R_{\max }^{\beta \geq A}$. For convenience, we define

$$
Q(x) \triangleq \exp \left(N_{0} / x\right) E_{1}\left(N_{0} / x\right) .
$$

substituting $\beta^{*}=B$ into $R_{\max }^{\beta \leq B}$, we get

$$
\begin{aligned}
R_{\max }^{\beta \leq B, \beta^{*}=B}= & \ln \left(g_{2 r}^{2}-\frac{g_{2 r}^{3}}{g_{1 r}} e^{\theta}\right)+2 \ln \left(\frac{P_{T}}{N_{0}}\right) \\
& -2 \lambda-Q\left(P_{c r}\right)-Q\left(P_{b}\right) .
\end{aligned}
$$

Similarly, we obtain

$$
\begin{aligned}
R_{\max }^{\beta \geq A, \beta^{*}=A}= & \ln \left(g_{1 r}^{2}-\frac{g_{1 r}^{3}}{g_{2 r}} e^{\theta}\right)+2 \ln \left(\frac{P_{T}}{N_{0}}\right) \\
& -2 \lambda-Q\left(P_{c r}\right)-Q\left(P_{b}\right)
\end{aligned}
$$

and

$$
\begin{aligned}
R_{\max }^{B \leq \beta \leq A, \beta^{*}=0.5}= & \ln \left(g_{1 r} g_{2 r}\right)+2 \ln \left(\frac{P_{T}}{N_{0}}\right) \\
& -2 \ln 2-2 \lambda-Q\left(P_{c r}\right)-Q\left(P_{b}\right) .
\end{aligned}
$$

Now we compare $R_{\max }^{\beta \leq B, \beta^{*}=B}$ and $R_{\max }^{B<\beta<A, \beta^{*}=0.5}$. Let $R_{\max }^{\beta \leq B, \beta^{*}=B}<R_{\max }^{B<\beta<A, \beta^{*}=0.5}$, after some manipulations, it leads to

$$
g_{2 r}^{2} e^{\theta}+g_{1 r}^{2} e^{-\theta-\ln 4}-g_{1 r} g_{2 r}>0
$$

We set $G_{2}=g_{2 r}^{2} e^{\theta}+g_{1 r}^{2} e^{-\theta-\ln 4}-g_{1 r} g_{2 r}$, then $G_{2}$ can be transformed into

$$
G_{2}=\left(g_{2 r}-\frac{g_{1 r}}{2 e^{\theta}}\right)^{2} e^{\theta}
$$

Since $e^{\theta}>0$, we get $G_{2} \geq 0$. That is to say, $R_{\max }^{B<\beta<A, \beta^{*}=0.5} \geq R_{\max }^{\beta \leq B, \beta^{*}=B}$ always stand up. The same way, we can verify that

$$
R_{\max }^{B<\beta<A, \beta^{*}=0.5} \geq R_{\max }^{\beta \geq A, \beta^{*}=A}
$$

Next, we compare the values of $R_{\max }^{\beta \geq A, \beta^{*}=A}$ and $R_{\max }^{\beta \leq B, \beta^{*}=B}$. The following results can be obtained

$$
\begin{cases}R_{\max }^{\beta \leq B, \beta^{*}=B}>R_{\max }^{\beta \geq A, \beta^{*}=A} & \text { if } g_{1 r}>g_{2 r} \\ R_{\max }^{\beta \leq B, \beta^{*}=B}<R_{\max }^{\beta \geq A, \beta^{*}=A} & \text { if } g_{1 r}<g_{2 r}\end{cases}
$$

Finally, we consider the value ranges of $A$ and $B$. Since $1-g_{2 r} e^{\theta} / g_{1 r}$, we have $B<1$. According to the analysis above, we get the following results for the case $G_{1} \geq 0$

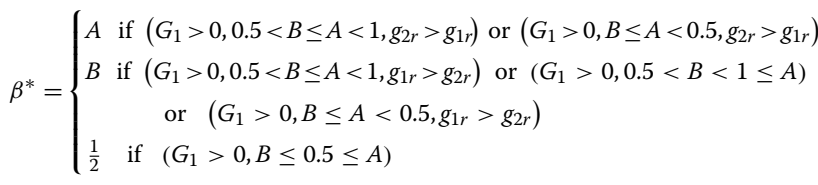

Having the above results and combining with (98), it yields the desired result.

\section{Funding}

This work was supported by the National Science Foundation (NSFC) for Distinguished Young Scholars of China with Grant 61625106 and the NSFC with Grant 61531011,61427801.

\section{Authors' contributions}

$\mathrm{HZ}$ and SJ conceived the study. YN, YW, and SJ are designed and modelled the study. YN, SJ, and K-KW are responsible for the performance analysis. YN, YW $\mathrm{K}-\mathrm{KW}$, and $\mathrm{HZ}$ are responsible for the results interpretation and manuscript writing: . All authors read and approved the final manuscript. 


\section{Competing interests}

The authors declare that they have no competing interests.

\section{Publisher's Note}

Springer Nature remains neutral with regard to jurisdictional claims in published maps and institutional affiliations.

\section{Author details}

${ }^{1}$ Jiangsu Normal University, Nanjing 210013, China. ${ }^{2}$ National Mobile Communications Research Laboratory, Southeast University, Nanjing 210096, China. ${ }^{3}$ Department of Electronic and Electrical Engineering, University College London, London, UK. ${ }^{4}$ Jiangsu Key Laboratory of Wireless Communications, Nanjing University of Posts and Telecommunications, Nanjing 210003, China.

Received: 10 January 2017 Accepted: 18 April 2017

Published online: 30 May 2017

\section{References}

1. IEEE. Standard 802.16e-2005. part16: Air interface for fixed and mobile broadband wireless access systems amendment for physical and medium access control layers for combined fixed and mobile operation in licensed band, (2005)

2. $3 G P P, T S 36.213 \vee$ 10.5.0. Physical layer procedures (Release 10) for Evolved Universal Terrestrial Radio Access (E-UTRA) (2012)

3. M Baker, From LTE-Advanced to the future. IEEE Commun. Mag. 50(2), 116-120 (2012)

4. TNakamura, S Nagata, A Benjebbou, et al., Trends in small cell enhancements in LTE Advanced. IEEE Commun. Mag. 51(2), 98-105 (2013)

5. 3GPP, RWS-120045. Summary of 3GPP TSG-RAN workshop on Release 12 and onward (2012)

6. F Boccardi, RW Heath Jr, A Lozano, TL Marzetta, P Popovski, Five disruptive technology directions for 5G. IEEE Commun. Mag. 52(2), 74-80 (2014)

7. YY Ni, J Shi, R Tian, KK Wong, HB Zhu, SX Shao, in Proc. IEEE WCNC. Outage analysis for device-to-device communication assisted by two-way decode-and-forward relaying, (Shanghai, 2013)

8. G Fodor, E Dahlman, G Mildh, S Parkvall, N Reider, G Miklos, Z Turanyi, Design aspects of network assisted device-to-device communications. IEEE Commun. Mag. 50(3), 170-177 (2012)

9. $3 G P P, T R 22.803 \vee 12.2 .0$. Feasibility study for Proximity Services (ProSe) (2013)

10. A Asadi, Q Wang, V Mancuso, A survey on device-to-device communication in cellular networks. IEEE Commun. Survey Tutor. 16(4), 1801-1809 (2014)

11. K Doppler, CH Yu, CB Ribeiro, P Jaanis, in Proc. IEEE WCNC. Mode selection for device-to-device communication underlaying an, LTE-advanced network, (Sydney, 2010)

12. M Jung, K Hwang, SY Choi, in Proc. IEEE VTC. Joint mode selection and power allocation for power-efficient device-to-device communication, (Yokohama, 2012)

13. P Janis, V Koivunen, C Ribeiro, J Korhonen, K Doppler, K Hugl, in Proc. IEEE VTC. Interference-aware resource allocation for device-to-device radio underlaying cellular networks, (Barcelona, 2009)

14. CH Yu, K Doppler, CB Ribeiro, C Wijting, K Hugl, O Tirkkonen, V Koivunen, Device-to-device communication underlaying cellular communications system. Int. J Commun. Netw. Syst. Sci. 47(12), 42-49 (2009)

15. K Doppler, MP Rinne, C Wijting, Device-to-device communication as an underlay to LTE-advanced networks. IEEE Commun. Mag. 47(12), 29-42 (2009)

16. CH Yu, O Tirkkonen, K Doppler, CB Ribeiro, in Proc. IEEE ICC. Power optimization of device-to-device communication underlaying cellular communication, (Dresden, 2009)

17. B Wang, L Chen, X Chen, X Zhang, D Yang, in Proc. IEEE VTC. Resource allocation optimization for device-to-device communication underlaying cellular networks, (Budapest, 2011)

18. CH Yu, K Doppler, CB Ribeiro, O Tirkkonen, Resource sharing optimization for device-to-device communication underlaying cellular networks. IEEE Trans. Wireless Commun. 10(8), 2752-2763 (2011)

19. $\vee$ der Meulen, Three-terminal communication channels. Adv. Appl. Prob. 3, 120-154 (1971)
20. JN Laneman, GW Wornell, DNC Tse, Cooperative diversity in wireless networks: Efficient protocols and outage behavior. IEEE Trans. Info. Theory. 50(12), 3062-3080 (2004)

21. H Bolcskei, RU Nabar, O Oyman, AJ Paulraj, Capacity scaling laws in MIMO relay networks. IEEE Trans. Wireless Commun. 5(6), 1433-1444 (2006)

22. JN Laneman, GW Wornell, Distributed space-time-coded protocols for exploiting cooperative diversity in wireless nertworks. IEEE Trans. Info. Theory. 49(10), 2415-2425 (2003)

23. A Sendonaris, E Erkip, B Aazhang, User cooperation diversity-Part I: System description. IEEE Trans. Commun. 51(11), 1927-1938 (2003)

24. A Sendonaris, E Erkip, B Aazhang, User cooperation diversity-Part II: Implementation aspects and performance analysis. IEEE Trans. Commun. 51(11), 1939-1948 (2003)

25. TM Cover, AE Gamal, Capacity theorems for the relay channel. IEEE Trans. Inform. Theory. 25, 572-584 (1979)

26. B Rankov, A Wittneben, Spectral efficient protocols for half duplex fading relay channels. IEEE J Sel. Areas Commun. 25(2), 379-389 (2007)

27. RY Louie, YH Li, B Vucetic, Practical physical layer network coding for two-way relay channels: Performance analysis and comparison. IEEE Trans. Wireless Commun. 9(2), 764-777 (2010)

28. M Hasan, E Hossain, Distributed resource allocation for relay-aided device-to-device communication: a message passing approach. IEEE Trans. Wireless Commun. 13(11), 6326-6341 (2014)

29. T Kim, MM Dong, An iterative approach to joint relay selection and resource allocation for D2D communications. IEEE Wireless Commun. Lett. 3(6), 625-628 (2014)

30. LF Wang, T Peng, YF Yang, WB Wang, in Proc. IEEE VTC. Interference constrained D2D communication with relay underlaying cellular networks, (Vegas, 2013)

31. G Huang, Y Wang, J Coon, in Proc. IEEE PacRim. Performance of multihop decode-and-forward and amplify-and-forward delay networks with channel estimation, (Quanzhou, 2007)

32. MM Molu, N Goertz, in Proc. ISWCS. Amplify and forward relaying; Channel model and outage behaviour, (Paris, 2012)

33. C Zhong, S Jin, KK Wong, Dual-hop systems with noisy relay and interference-limited destination. IEEE Trans. Commun. Soc. 58(3), 764-768 (2010)

34. L Wang, $\mathrm{H}$ Jin, XD Ji, Y Li, MG Peng, in Proc. IEEE MAPE. Power allocation for cognitive D2D communication assisted by two-way relaying, (Chengdu, 2013)

35. L Sun, QH Du, PY Ren, YC Wang, Two birds with one stone: Towards secure and interference-free D2D transmission via constellation rotation. IEEE Trans. Veh. Technol. 65(10), 8767-8774 (2010)

36. DS Gurjar, PK Upadhyay, in Proc. IEEE TENCON. Three-phase overlay D2D communications in traffic-aware two-way cellular systems, (Marina Bay Sands, 2016)

37. YY Pei, YC Liang, Resource allocation for device-to-device communications overlaying two-way cellular networks. IEEE Trans. Wireless Commun. 12(7), 3611-3621 (2013)

38. Y Yu, in Proc. IEEE ISIT. Broadcasting when receivers know some message a priori, (Nice, 2007)

39. XS Liang, S Jin, XQ Gao, KK Wong, Outage performance for decode-and-forward two-way relay network with multiple interferers and noisy relay. IEEE Trans. Commun. 61(2), 521-531 (2013)

40. XS Liang, S Jin, XQ Gao, KK Wong, C Sun, in Proc. IEEE GLOBECOM. Outage Performance for Interference-Limited Decode-and-Forward Two-Way Relaying Networks, (California, 2012), pp. 4554-4559

41. V Raghavan, SV Hanly, W Veeravalli, Statistical beamforming on the grassmann manifold for the two-user broadcast channel. IEEE Trans. Inform. Theory. 59(10), 6464-6489 (2013)

42. J Zhang, JG Andrews, Adaptive spatial intercell interference cancellation in multicell wireless networks. IEEE J. Select. Areas Commun. 28(9), 1455-1468 (2010)

43. IS Gradshteyn, IM Ryzhik, Table of Integrals, Series, and Products, 6th ed. (Academic Press, San Diego, California, 2000) 